\title{
Journal contacts
}

\section{ABOUT THE JOURNAL}

Acta IMEKO is an e-journal reporting on the contributions on the state and progress of the science and technology of measurement. The articles are mainly based on presentations presented at IMEKO workshops, symposia and congresses. The journal is published by IMEKO, the International Measurement Confederation. The ISSN, the international identifier for serials, is 2221-870X.

\section{EDITOR-IN-CHIEF}

Dušan Agrež, Slovenia

Leopoldo Angrisani, Italy Filippo Attivissimo, Italy Eulalia Balestieri, Italy

Eric Benoit, France

Paolo Carbone, Italy

Lorenzo Ciani, Italy

Catalin Damian, Romania

Pasquale Daponte, Italy

Luca De Vito, Italy

Luigi Ferrigno, Italy

Edoardo Fiorucci, Italy

Alistair Forbes, United Kingdom

Helena Geirinhas Ramos, Portugal

Sabrina Grassini, Italy

Fernando Janeiro, Portugal

Konrad Jedrzejewski, Poland

Andy Knott, United Kingdom

\section{FOUNDING EDITOR-IN-CHIEF}

Paul P. L. Regtien, Netherlands

\section{EDITORIAL BOARD}

Francesco Lamonaca, Italy

Massimo Lazzaroni, Italy

Fabio Leccese, Italy

Rosario Morello, Italy

Michele Norgia, Italy

Pedro Miguel Pinto Ramos, Portugal

Nicola Pompeo, Italy

Sergio Rapuano, Italy

Dirk Röske, Germany

Alexandru Salceanu, Romania

Constantin Sarmasanu, Romania

Lorenzo Scalise, Italy

Emiliano Schena, Italy

Enrico Silva, Italy

Krzysztof Stepien, Poland

Marco Tarabini, Italy

\section{ASSOCIATE EDITOR}

Dirk Röske, Germany

\section{SECTION EDITORS (Vol. 7 and 8)}

Marcantonio Catelani, Italy

Lorenzo Ciani, Italy

Egidio De Benedeto, Italy

Alessandro Germak, Italy

Sabrina Grassini, Italy

Konrad Jędrzejewski, Poland

Min-Seok Kim, Korea

Fabio Leccese, Italy

Rosario Morello, Italy

Vilmos Palfi, Hungary

Nicola Pasquino, Italy

Franco Pavese, Italy

Alexandru Salceanu, Romania

Jan Saliga, Slovakia

Jorge C. Torres-Guzman, Mexico

Ian Veldman, South Africa

Claudia Zoani, Italy

Alessandro Depari, Italy

Emiliano Sisinni, Italy

\section{ABOUT IMEKO}

The International Measurement Confederation, IMEKO, is an international federation of actually 41 national member organisations individually concerned with the advancement of measurement technology. Its fundamental objectives are the promotion of international interchange of scientific and technical information in the field of measurement, and the enhancement of international co-operation among scientists and engineers from research and industry.

\section{ADDRESSES}

\begin{tabular}{ll}
\hline Principal Contact & Prof. Dušan Agrež \\
& University of Ljubljana \\
& Faculty of Electrical Engineering \\
& Tržaška 25, 1000 Ljubljana, Slovenia \\
& e-mail: $\underline{\text { dusan.agrez@,fe.uni-li.si }}$ \\
& \\
ACTA IMEKO & Alessandro Depari, Emiliano Sisinni \\
& Department of Information Engineering \\
& University of Brescia \\
& Via Branze 38 - 25123, Brescia, Italy \\
& e-mail: alessandro.depari@unibs.it, emiliano.sisinni@unibs.it \\
\hline
\end{tabular}

Support Contact

Dr. Dirk Röske

Physikalisch-Technische Bundesanstalt (PTB)

Bundesallee 100, 38116 Braunschweig, Germany

e-mail: dirk.roeske@ptb.de 